\section{Extreme weather events}

SIR - The seasonally recurring oceanic El Niño phenomenon is associated with extreme weather conditions ${ }^{1}$. Positive phases of the tropical sea-surface temperature oscillation have been connected with unusually rainy weather over the southwestern United States, while the negative phases, usually referred to as La Niña, have been plausibly associated with extreme dry conditions over the central United States.

Since the oceanic oscillation was first connected by dynamic and thermodynamic considerations ${ }^{2}$ to the atmospheric Southern Oscillation, understanding of the coupled ocean-atmosphere El Niño/ Southern Oscillation (ENSO) phenomenon has increased dramatically ${ }^{1}$, leading to hopes that these events could be predicted a year or so in advance. Statistical prediction models of $\mathrm{ENSO}^{3}$ - based on the analysis of sea-surface temperature and surface wind fields and dynamical models ${ }^{4}$ can forecast events with some confidence out to 6 months or even 1 year.

These models try to forecast the detailed geographical distribution of oceanic and atmospheric variables affected by the ENSO cycle. This is a more difficult task than telling whether an El Niño or La Niña event will occur; univariate indicators of ENSO can be helpful for the latter. The Southern Oscillation index computed from time series of sea-level pressure at Tahiti and Darwin, Australia, has good diagnostic value. Minima in this index are associated with El Niño events, whereas maxima correspond to La Niña events ${ }^{1}$.

A combination of singular-spectrum analysis $^{5}$ and the maximum entropy method $^{6}$ seems to hold promise for predicting the ENSO cycle 2-3 years in advance. Singular-spectrum analysis is a variant of principal component analysis applied in the time domain; it filters out variability unrelated to ENSO, separating the quasi-biennial 2-3-year variability from a lower-frequency 4-6-year El Niño-La Niña cycle; the total variance associated with ENSO combines the quasi-biennial and lower-frequency

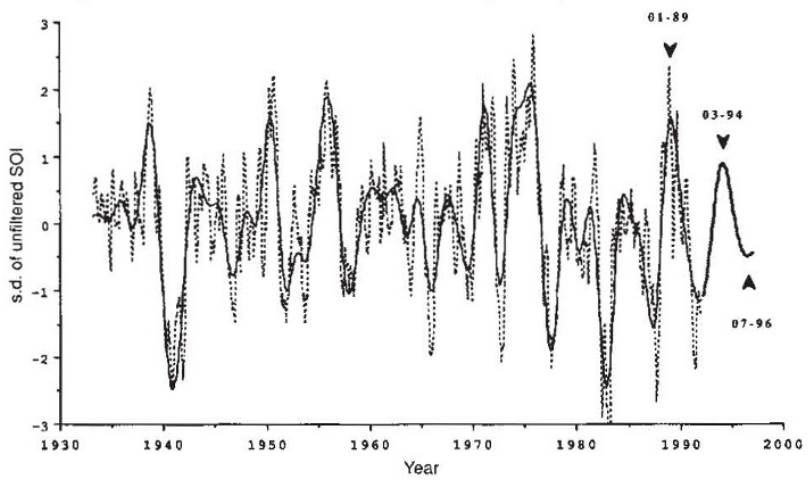

Southern Oscillation index series obtained by singularspectrum analysis (solid, thin line); 5-month running mean of the index (dotted, thin line); and forecast obtained by applying the maximum-entropy method to the leading temporal principal components of the index (solid, thick line).

\section{Cosmic microwave background}

SIR - Fluctuations of the cosmic microwave background from the COBE satellite (discussed recently in News and Views ${ }^{1}$ ) have been widely interpreted as providing confirmatory evidence for the Big Bang explanation of this radiation, the fluctuations being taken to represent the signatures of galaxy formation in the early Universe. Alternative explanations for the microwave background, involving thermalization, can also account for the observed energy density of the radiation and can also produce intensity fluctuations of the kind that were observed by COBE.

For such a model involving thermalization by iron whiskers of diameter $\sim 0.02 \mu \mathrm{m}$ and length $\sim 1 \mathrm{~mm}$, which possess a flat absorption curve over the wavelength range $\sim 0.2 \mathrm{~mm} \simeq 2 \mathrm{~cm}$, with an average mass absorption coefficient of $1.7 \times 10^{7} \mathrm{~cm}^{2} \mathrm{~g}^{-2}$ and a smoothed-out cosmological mass density of $10^{-34} \mathrm{~g} \mathrm{~cm}^{-3}$, the effective 'last emission' photosphere is an Earth-centred spherical surface of radius $R \simeq 200 \mathrm{mpc}$ (refs 2-4). Fluctuations in the density distribution of iron needles in the foreground at distances nearer than $200 \mathrm{mpc}$, which are inevitable, would naturally lead to a microwave extinction which varies in intensity across the sky, but which does not alter the spectrum, leading in turn to fluctuations in the measured microwave background temperature. Some of these fluctuations could arise from clouds confined to the Galaxy, others could be of extragalactic origin.

With a reasonable assumption of density fluctuations of the order of 1 part in 10,000 occurring over inter-cluster distance scales of $L \simeq 20 \mathrm{mpc}$, the resulting temperature fluctuations can be shown to be $\Delta T / T \simeq 5 \times 10^{-6}$, as observed.

A typical angular scale of fluctuations would be $\Delta \theta \simeq L / R \simeq 6^{\circ}$, but larger fluctuations up to $90^{\circ}$ could, of course, arise less frequently from a few local clouds. The Big Bang explanation of the latest $\mathrm{COBE}$ result is thus not unique, and alternative thermalization models cannot be excluded.

\section{N. C. Wickramasinghe}

School of Mathematics,

University of Wales College Cardiff,

Senghenydd Road,

Cardiff CF2 4AG, UK

1. Silk, J. Nature 356, 741-742 (1992).

2. Hoyle, F. \& Wickramasinghe, N. C. Astrophys. Space Sci. 147, 245-256 (1988).

3. Wickramasinghe, N. C. Wickramasinghe, A.N. \& Hoyle, F. Astrophys. Space Sci. (in the press).

4. Wickramasinghe, N. C. Astrophys. Space Sci. (in the press). 